\title{
Aspectos biológicos de Zabrotes subfasciatus (Bohemann, 1833) (Coleoptera, Bruchidae) em Phaseolus vulgaris L., cv. Carioca (Fabaceae), sob condições de laboratório ${ }^{1}$
}

\author{
Lisiane Taiatella Sari ${ }^{2,3}$ \\ Cibele Stramare Ribeiro-Costa ${ }^{2,3}$ \\ Paulo Roberto Valle da Silva Pereira ${ }^{4}$
}

\begin{abstract}
Biological aspects of Zabrotes subfasciatus (Bohemann, 1833) (Coleoptera, Bruchidae) on Phaseolus vulgaris L., cv. Carioca (Fabaceae), under laboratory conditions. Zabrotes subfasciatus is a serious pest of common beans, P. vulgaris L.. In Brazil there are several studies dealing with resistance of bean genotypes to this insect, while other studies have emphasized the utilization of oils and powders from plants to repel their attack. In this paper, fecundity, fertility, pattern of oviposition, life cycle and longevity were evaluated for a Brazilian stock from the Goiás State on P. vulgaris cv. Carioca, at $30^{\circ} \mathrm{C}$ and $70 \%$ R.H. The mean fecundity was 38 eggs per female and $73 \%$ of viability. Egg laying showed an aggregated pattern. Males and females lived an average of 13 and 9 days, respectively. The total life cycle lasted for about 28 days.
\end{abstract}

KeYwords. Phaseolus vulgaris; stored bean pest; Zabrotes subfasciatus.

\section{INTRODUÇÃO}

Cerca de 20 espécies pertencentes a seis gêneros de Bruchidae desenvolvem-se em grãos de leguminosas armazenados e consumidos pelo homem (SouthGate 1979). O caruncho-do-feijão, Zabrotes subfasciatus (Boheman, 1833) é originária das regiões tropicais e subtropicais das Américas Central e do Sul e é uma das principais pragas do feijão, Phaseolus vulgaris L. (Fabaceae), durante o armazenamento (Dendy \& Credland 1991; Haines 1991). A espécie tem sido introduzida em muitos países da Europa por meio de feijões infestados, onde tem ocasionalmente ampliado seu registro de hospedeiros, tornando-se também uma praga séria de outros legumes (MеIк \& Dobie 1986).

A fêmea oviposita diretamente nas sementes após a deiscência das vagens ou pode infestar as sementes ainda dentro das mesmas, utilizando-se de perfurações realizadas por outros insetos (CRedLAND \& Dendy 1992). Ao contrário da maioria dos bruquídeos, as fêmeas necessitam do contato com a semente para estimular a ovogênese (PIMBERT \& PIERRE 1983;
Pimbert 1985a, c). Após a eclosão, a larva perfura o tegumento da semente e completa o seu desenvolvimento consumindo, apenas, o conteúdo de uma semente (Southgate 1979; CREDland \& Dendy 1992). Em armazenamento, os adultos podem produzir muitas gerações, sem se alimentarem (JoHNSON 1989).

Os prejuízos na pós-colheita traduzem-se em uma considerável redução de peso, diminuição da qualidade nutricional, declínio do poder germinativo das sementes, depreciação comercial devido à presença de insetos adultos ou imaturos, fragmentos e excrementos. A estes prejuízos somam-se os danos indiretos, pelo favorecimento da entrada de ácaros e microrganismos, principalmente fungos, que contribuem para o aquecimento dos grãos (HoHMANN \& CARValHo 1989).

Zabrotes subfasciatus tem sido amplamente utilizada em testes de resistência de cultivares e linhagens de P. vulgaris no Brasil (OliveIRA et al. 1979; Oriani et al. 1996; WANDERLEY et al. 1997; LARA 1997; MAZZONETTO \& BoIçA Jr. 1999). Outros estudos com esta espécie enfocam seu controle através da

1. Contribuição $n^{\circ} 1453$ do Departamento de Zoologia, Universidade Federal do Paraná.

2. Departamento de Zoologia, Universidade Federal do Paraná. Caixa Postal 19020, 81531-980 Curitiba-PR, Brasil. Endereço eletrônico: lisi@bio.ufpr.br e stra@ufpr.br

3. Bolsistas do CNPq

4. EMBRAPA Roraima - Entomologia. Caixa Postal 133,69301-970 Boa Vista-RR, Brasil. Endereço eletrônico: paulo@cpafrr.embrapa.br 
utilização de óleos essenciais ou pós vegetais (Oliveira \& VendRAmim 1999; Weaver et al. 1994). Poucos trabalhos foram realizados com a finalidade de investigar os parâmetros biológicos de diferentes populações de Z. subfasciatus (MEIK \& Dobie 1986; Pimbert 1985b; Dendy \& CRedland 1991; Credland \& Dendy 1992). Essa pesquisa é considerada de fundamental importância na determinação do potencial da praga, podendo auxiliar na previsão da resposta dessas populações a novas cultivares de feijão. Dessa forma, a presente pesquisa visou determinar alguns aspectos biológicos de uma população brasileira de Z. subfasciatus.

\section{MATERIAL E MÉTODOS}

Os adultos de Z. subfasciatus foram obtidos junto à EMBRAPA, Centro Nacional de Pesquisa de Arroz e Feijão, em Goiás. A criação-estoque da EMBRAPA foi mantida em temperatura ambiente, tendo início em 1994 com sementes de $P$. vulgaris, cultivar Goiano Precoce e, após três anos, foi alterada para a cultivar Jalo Precoce; ambas as cultivares são do grupo Manteigão, de ciclo precoce e grãos graúdos. Adultos introduzidos na criação foram provenientes de $P$. vulgaris coletados em armazéns na região de Goiânia (Massaru Yokoyama, comunicação pessoal).

A criação-estoque, proveniente da EMBRAPA, foi mantida à temperatura ambiente, no hospedeiro $P$. vulgaris $\mathrm{cv}$. Carioca, no Departamento de Zoologia, da Universidade Federal do Paraná. Dentre os feijões de cor, esta cultivar destaca-se pela alta produtividade e importância comercial (ModA-CiRINo et al. 1989).

A partir da terceira geração foram selecionados grãos com posturas, mantendo-se apenas um ovo sobre cada grão. Esses grãos foram isolados em cápsulas de gelatina até a emergência dos adultos. Uma a duas horas após a emergência foram formados 30 casais, acondicionados em recipientes de vidro (10 cm de altura x $1,5 \mathrm{~cm}$ de diâmetro) com tampa e rosca, contendo seis grãos de $P$. vulgaris, cv. Carioca, com teor de umidade de $14 \%$, os quais foram retirados de embalagens plásticas de $1 \mathrm{Kg}$ comercializadas em Curitiba, Paraná, safra de 1999. O experimento foi mantido em câmara climatizada a $30^{\circ} \mathrm{C} \pm$ $1{ }^{\circ} \mathrm{C}$, fotofase $12 \mathrm{~h}$ e $65 \pm 5 \% \mathrm{UR}$.

Para a determinação da fecundidade, a cada 24 horas, os casais foram transferidos para recipientes semelhantes aos descritos anteriormente, contendo seis grãos não infestados. A cada transferência, foi registrado o número de ovos por fêmea e por grão. Este procedimento foi repetido até a morte das fêmeas.

Para a determinação do tipo de dispersão dos ovos nos grãos, foram utilizados o índice de Morisita (1962) e o índice médio de agregação (DENDY \& CREDLAND 1991), avaliando-se a postura diária de cada fêmea em seis grãos, a partir dos dados obtidos no período de maior oviposição.

\section{RESULTADOSEDISCUSSÃO}

O período de pré-oviposição foi de 1,2 $\pm 0,71$ dias e o de pós-oviposição de 1,2 $\pm 1,10$ dias. Pajni \& Jabbal (1986) observaram que machos e fêmeas recém-emergidos de grãos de Phaseolus lunatus (Rajmash), geralmente, são capazes de copular após uma hora e as fêmeas, de ovipositar de duas a 30 horas após a cópula, nas mesmas condições desse experimento $\left(30^{\circ} \mathrm{Ce} 70 \%\right.$ UR).

As fêmeas ovipositaram durante 5,93 $\pm 0,96$ dias, valor próximo (7 dias) ao registrado por PAJNI \& JABBAL (1986). Os mesmos autores analisaram o efeito da temperatura, 25, $30 \mathrm{e}$ $35^{\circ} \mathrm{C}$ e umidade relativa, 50,70 e $90 \%$, na duração do período de oviposição e verificaram uma tendência de aumento do número de dias e diminuição do número de ovos/fêmea/dia na temperatura mais baixa, independente da influência da umidade. HowE \& CuRRIE (1964), também verificaram essa tendência, em diferentes temperaturas e mesma umidade (70\%).

O período de maior oviposição compreendeu o $3^{\circ}$ e $4^{\circ}$ dias após a emergência e acasalamento dos adultos (Fig. 1), sendo a freqüência de oviposição entre 5 e 15 ovos por fêmea. Carvalho \& Rossetto (1968), em experimento realizado em condições semelhantes, $30-32^{\circ} \mathrm{C} \mathrm{e} 70-75 \%$ UR, com a cv. Pintado de $P$. vulgaris, registraram o pico de oviposição no $5^{\circ}$ dia.

A dispersão dos ovos nos grãos, para os dois índices utilizados, foi agregada, observando-se sempre grãos sem postura (Tabela I). DenDy \& CREDLAND (1991) relataram o mesmo comportamento a $27^{\circ} \mathrm{Ce} 70 \%$ UR, na cv. de feijão "Red Kidney"; mas, como as avaliações dos autores não foram diárias, não foi possível afirmar se a fêmea ovipositou em um mesmo grão em uma única vez ou em diversas ocasiões. Entretanto, a presente pesquisa indica, em função da avaliação diária, que os ovos são colocados agrupados, de uma só vez. PIMBERT (1985c), ao realizar um experimento com cinco grãos enfileirados de Phaseolus vulgaris cv. Canário, observou uma heterogeneidade no comportamento de oviposição, ou seja, $54 \%$ das fêmeas de Z. subfasciatus depositaram ovos de forma agregada, enquanto o restante de forma aleatória.

O número médio de ovos por grão foi de $1,94 \pm 1,17$, variando

Tabela I. Valores diários de fecundidade (no de ovos/fêmea/dia) e dispersão de ovos de Zabrotes subfasciatus em grãos de Phaseolus vulgaris, c.v. Carioca, a $30 \pm 1{ }^{\circ} \mathrm{C}, 65 \pm 5 \%$ UR.

\begin{tabular}{ccccc}
\hline Hospedeiro & $\mathrm{n}^{\circ}$ de sementes/dia & $\mathrm{n}^{\mathrm{o}}$ ovos/fêmea/dia & Índice de Morisita $\left(\mathrm{I}_{\delta}\right)$ & Índice médio de agregação \\
\hline P. vulgaris & 6 & 6,39 & $1,255^{*}$ & $1,898^{* *}$ \\
\hline
\end{tabular}

$* \mathrm{I}_{\delta}>1$ indica agregação, valor obtido é significativo pelo teste $\mathrm{F}$ a $1 \%$ de probabilidade.

** valores maiores que 1 indicam agregação. 


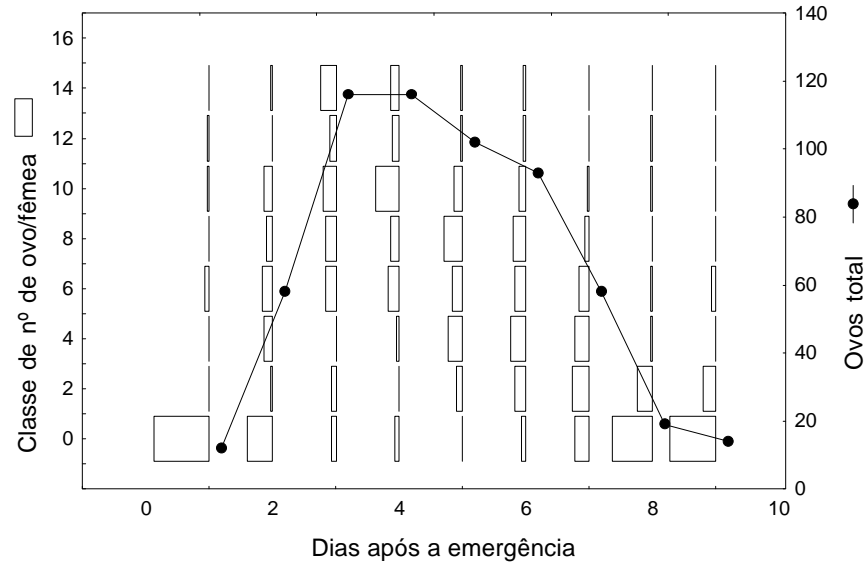

Fig. 1. Número total de ovos/dia e frequência de ovos/fêmea/dia depositados por 30 fêmeas de Zabrotes subfasciatus desde a emergência e acasalamento até o final do estágio adulto, em avaliações a cada 24 horas, em grãos de Phaseolus vulgaris, variedade Carioca, a $30 \pm 1^{\circ} \mathrm{C}$ e $65 \pm 5 \%$ UR.

de 1 até 10 ovos, com 96,3\% dos grãos contendo de 1 a 5 ovos (Fig. 2).

A fecundidade média de $Z$. subfasciatus foi de 38,13 $\pm 9,63$ ovos por fêmea, valor próximo aos obtidos por FERREIRA (1960) $\left(27^{\circ} \mathrm{C}, 75 \% \mathrm{UR}, \mathrm{cv}\right.$. Manteiga de P. vulgaris $)$ e Howe \& CuRRIE (1964) $\left(30^{\circ} \mathrm{C}, 70 \% \mathrm{UR}\right.$ cv. Haricot), ou seja, aproximadamente 44,4 e 35,5 ovos por fêmea, respectivamente. Outros autores encontraram valores superiores, como Dendy \& CREDland (1991) que obtiveram, em média, 55 ovos por fêmea, PAJNI \& JABBAL (1986) que relataram cerca de 52 ovos por fêmea a $30^{\circ} \mathrm{C}$ e 70\% UR, na cv. Rajmash de Phaseolus lunatus e Goloв \& KiLMINSTER (1982) com 50, $7 \pm 1,7$ ovos por fêmea a $25^{\circ} \mathrm{Ce} 70 \%$ UR na cv. Diacol-Calima. CARvalho \& Rossetto (1968) verificaram menor valor médio, ou seja, 22 ovos por fêmea.

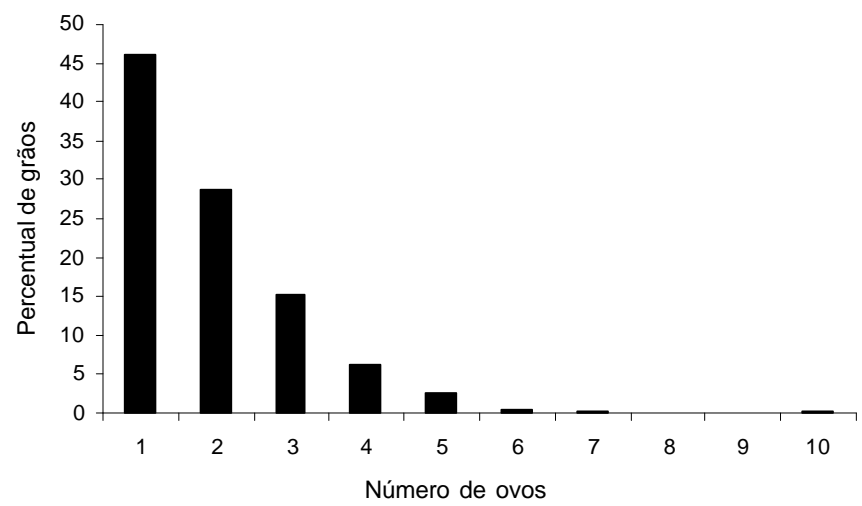

Fig. 2. Percentual de grãos e número de ovos depositados por 30 fêmeas de Zabrotes subfasciatus desde a emergência e acasalamento até o final da fase de adulto, em avaliações a cada 24 horas, em seis grãos de Phaseolus vulgaris, c.v. Carioca, a $30 \pm 1^{\circ} \mathrm{C}$ e $65 \pm 5 \%$ UR.
Neste estudo observou-se um máximo de oito adultos emergindo da mesma semente, porém, nos trabalhos de PAJNI \& JabBAL (1986) e Dendy \& CREDLAND (1991) ocorreram casos onde mais de vinte adultos emergiram de uma única semente. $\mathrm{O}$ número de adultos que emergem também está relacionado ao tamanho das sementes ofertadas.

Credland \& Dendy (1992), estudando diferentes populações de $Z$. subfasciatus em cultivares de P. vulgaris $\left(27,1^{\circ} \mathrm{C}\right.$ e $70 \pm 10 \%$ UR $)$, observaram variações na fecundidade média (36,11 a 57,78 ovos por fêmea), no percentual de ovos eclodidos (de 89,7 a 99,6\%), na emergência média de adultos por fêmea (de 29,2 a 52,3) e no ciclo evolutivo (de 33,4 a 37,6 dias).

Com relação à fertilidade, verificou-se que $72,9 \%$ do total de ovos foram viáveis, gerando $50,4 \%$ de fêmeas. Muitos autores obtiveram valores de fertilidade também altos, mesmo a temperaturas diferentes: FERREIRA (1960), observou 90\% de ovos férteis a $27^{\circ} \mathrm{C}$; Howe \& CURRIE (1964), $78 \%$ a $30^{\circ} \mathrm{C}$; PAJNI \& JABBAL (1986), de 85 a 90\%, a 30 ${ }^{\circ} \mathrm{C}$; Golob \& KiLMINSTER (1982), $91,3 \%$ a $25^{\circ} \mathrm{Ce}$ Dendy \& CREDLAND (1991), $80 \%$ a $27^{\circ} \mathrm{C}$. CARVALHO $\&$ RossetTo (1968) obtiveram um valor mais baixo, $17,42 \%$ de ovos férteis a $30-32^{\circ} \mathrm{C}$ e $70-75 \%$ UR.

A longevidade dos adultos foi de 9,4 $\pm 1,54$ dias para as fêmeas e 13,3 $\pm 2,51$ dias para os machos e o ciclo evolutivo foi de 28,9 $\pm 8,5$ dias. Golob \& Kilminster (1982) encontraram o valor médio de 12,8 $\pm 0,1$ dias para a longevidade das fêmeas. CARDONA et al. (1989) e DeNDY \& CREDLAND (1991) obtiveram resultados semelhantes para o ciclo evolutivo, com 34 dias.

A correlação entre o período de oviposição, o número total de ovos/fêmea e a emergência de adultos foi positiva (Fig. 3), evidenciando que quanto maior o período de oviposição, maior o número de ovos, e que os ovos das fêmeas com maior período de oviposição geram um maior número de adultos. Desta maneira, pode-se observar que apesar das fêmeas concentrarem

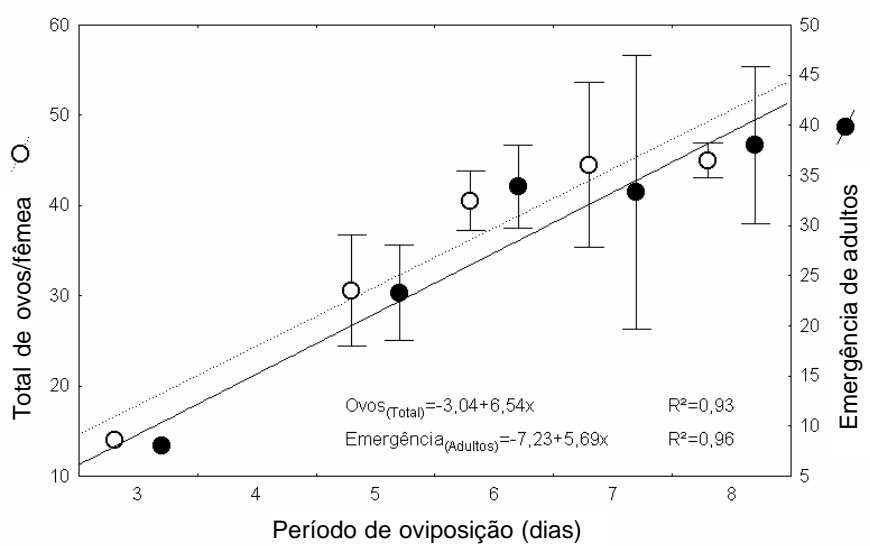

Fig. 3. Relação entre o número de ovos depositados por 30 fêmeas desde a emergência e acasalamento até o final da fase de adulto, período de oviposição e emergência de adultos de Zabrotes subfasciatus, em avaliações a cada 24 horas, em grãos de Phaseolus vulgaris, c.v. Carioca, a $30 \pm 1^{\circ} \mathrm{C}$, $65 \pm 5$ UR. 
a oviposição nos $3^{\circ} \mathrm{e} 4^{\circ}$ dias após a emergência e acasalamento (Fig. 1), a duração do período de oviposição é que determina o número de adultos a emergirem.

Agradecimentos. Ao Dr. Massaru Yokoyama, pesquisador da EMBRAPA, Centro Nacional de Pesquisa de Arroz e Feijão pelo envio de exemplares de Z. subfasciatus, ao Dr. Moisés Cordeiro Mourão de Oliveira Júnior, pesquisador da EMBRAPA Roraima, pelo auxílio na avaliação estatística e à Prof. Dra. Sonia Maria Noemberg Lázzari, pesquisadora da Universidade Federal do Paraná, pela revisão do manuscrito.

\section{REFERÊNCIAS}

Cardona, C.; C. E. Posso; J. Kornegay; J. Valor \& M. Serrano. 1989. Antibiosis effects of wild dry bean accessions on the Mexican bean weevil (Coleoptera: Bruchidae). Journal of Economic Entomology 82: 310-315.

Carvalho, R. P. L. De \& C. J. Rosseto. 1968. Biology of Zabrotes subfasciatus (Boheman) (Coleoptera: Bruchidae). Revista Brasileira de Entomologia 13: 195-197.

Credland, P. F. \& J. Dendy. 1992. Intraespecific variation in bionomic characters of the Mexican bean weevil, Zabrotes subfasciatus. Entomologia Experimentalis Applicata 65: 39-47.

Dendy, J. \& P. F. Credland. 1991. Development, fecundity and egg dispersion of Zabrotes subfasciatus. Entomologia Experimentalis Applicata 59: 9-17.

Ferreira, A. M. 1960. Subsídios para o estudo de uma praga do feijão (Zabrotes subfasciatus Boh. Coleoptera, Bruchidae) dos climas tropicais. Garcia de Orta Série de Estudos Agronomicos 8(3): 559-581.

Golob, P. \& A. Kilminster. 1982. The biology and control of Zabrotes subfasciatus (Boheman) (Coleoptera: Bruchidae) infesting red kidney beans. Journal of Stored Products Research 18: 95-101.

HAINES, C. P. 1991. Insects and arachnids of tropical stored products: Their biology and identification. $2^{a}$ edição. Kent, Natural Resources Institute, $246 \mathrm{p}$.

Hohmann, C. L. \& S. M. Carvalho. 1989. Pragas e seu controle, p. 217246. In: S. M. Carvalho; R. G. Carneiro; E. J. Mariot; L. C. Assumpção; R. P. L. Junior; L. R. Pereira; D. R. S. Siqueira; R. Hauagge \& S. R. Postiglioni (Eds.). O Feijão no Paraná. Londrina, IAPAR, Circular 63, $303 \mathrm{p}$.

Howe, R. W. \& J. E. CurRIE. 1964. Some laboratory observations on the rates of development, mortality and oviposition of several species of Bruchidae breeding in stored pulses. Bulletin of Entomological Research 55: 437-477.

Johnson, C. D. 1989. Adaptive radiation of Acanthoscelides in seeds: examples of legume-bruchid interactions. C. H. Stirton \& J. L. Zarucchi (eds.). Advances in legume biology. Monographs in Systematic Botany 29: 747-779.

LARA, F. M. 1997. Resistance of wild and near isogenic bean lines with arcelin variants to Zabrotes subfasciatus (Boheman). I - Winter crop. Anais da Sociedade Entomológica do Brasil 26(3): 551560 .

Mazzonetto, F. \& A. L. Boiça Jr. 1999. Determinação dos tipos de resistência de genótipos de feijoeiro ao ataque de Zabrotes subfasciatus (Boh.) (Coleoptera: Bruchidae). Anais da Sociedade Entomológica do Brasil 28(2): 307-311.

Mегк, J. \& P. DoвiE. 1986. The ability of Zabrotes subfasciatus to attack cowpeas. Entomologia Experimentalis Applicata 42: 151-158.

Moda-Cirino, V.; W. M. Kranz; M. A. Lollato; L. Oliari \& P. G. Ribeiro. 1989. Escolha e zoneamento de cultivares, p. 43-52. In: S. M. Carvalho; R. G. Carneiro; E. J. Mariot; L. C. Assumpção; R. P. L. Junior; L. R. Pereira; D. R. S. Siqueira; R. Hauagge \& S. R. Postiglioni (Eds.). O Feijão no Paraná. Londrina, IAPAR, Circular 63, 303 p.

Morisita, M. 1962. Id index, a measure of dispersion of individuals. Researches on Population Ecology 4: 1-7.

Oliveira, A. M.; C. E. Pacova \& S. Sudo. 1979. Incidência de Zabrotes subfasciatus Bohemann, 1833 e Acanthoscelides obtectus Say, 1831 em diversas cultivares de feijão armazenado (Coleoptera: Bruchidae). Anais da Sociedade Entomológica do Brasil 8(1): 45-55.

Oliveira, A. M. \& J. D. Vendramim. 1999. Repelência de óleos essenciais e pós vegetais sobre adultos de Zabrotes subfasciatus (Boh.) (Coleoptera: Bruchidae) em sementes de feijoeiro. Anais da Sociedade Entomológica do Brasil 28(3): 549-555.

Oriani, M. A; F. M. Lara \& A. L. BoiçA Jr. 1996. Resistência de genótipos de feijoeiro a Zabrotes subfasciatus (Boh.) (Coleoptera: Bruchidae). Anais da Sociedade Entomológica do Brasil 25(2): 213-216.

PAJNI, H. R. \& A. JABBAL. 1986. Some observations of Zabrotes subfasciatus (Boh.) (Bruchidae: Coleoptera). Research Bulletin of the Panjab University Science 37: 11-16.

Pimbert, M. 1985a. A model of host plant change of Zabrotes subfasciatus Boh. (Coleoptera: Bruchidae) in a traditional bean cropping system in Costa Rica. Biological Agriculture \& Horticulture 3: 39-54.

PimBert, M. 1985b. Reproduction and ovipositiion preferences of Zabrotes subfasciatus stocks reared from two host plant species. Entomologia Experimentalis Applicata 38: 273-276.

PimBerT, M. 1985c. Comparaison du comportement de ponte de Zabrotes subfasciatus Boh. (Col., Bruchidae) en présence de gousses ou de graines de Phaseolus vulgaris L. Biology of Behaviour 10: 309319.

Pimbert, M. \& D. Pierre. 1983. Ecophysiological aspects of bruchid reproduction. I. The influence of pod maturity and seeds of Phaseolus vulgaris and the influence of insemination on the reproductive activity of Zabrotes subfasciatus. Ecological Entomology 8: 8794

Southgate, B. J. 1979. Biology of the Bruchidae. Annual Review of Entomology 24(1): 449-473.

Wanderley, V. S; J. V. Oliveira \& M. L. Andrade Jr. 1997. Resistência de cultivares e linhagens de Phaseolus vulgaris L. a Zabrotes subfasciatus (Boh) (Coleoptera: Bruchidae). Anais da Sociedade Entomológica do Brasil 26 (2): 315-320.

Weaver, D. K.; F. V. Dunkel; R. C. Potter \& L. Netzurubanza. 1994. Contact and fumigant efficacy of powdered and intact Ocimum canum Sims (Lamiales: Lamiaceae) against Zabrotes subfasciatus (Bohemann) adults (Coleoptera: Bruchidae). Journal of Stored Products Research 30: 243-252. 